\title{
Newer forms of conventional ventilation for preterm newborns
}

\author{
Sunil K Sinha (Sunil.sinha@stees.nhs.uk)', Steven M Donn²
}

1.Professor of Paediatrics and Neonatal Medicine, University of Durham and James Cook University Hospital, Middlesbrough, TS4 3BW, United Kingdom

2.Professor of Pediatrics, Chief, Division of Neonatal-Perinatal Medicine, University of Michigan Health System, Ann Arbor, Michigan, USA

\section{Keywords}

Mechanical ventilation, Newborn, Newborn and

infant, Newborn babies

\section{Correspondence}

Sunil Sinha, University of Durham and

James Cook University Hospital, Middlesbrough,

TS4 3BW, United Kingdom.

Tel/fax: +44 (0) 1642854874 |

Email: Sunil.sinha@stees.nhs.uk

\section{Received}

8 February 2008; revised 14 April 2008;

accepted 18 April 2008.

DOI:10.1111/j.1651-2227.2008.00869.x

\section{INTRODUCTION}

Despite a recent increase in non-invasive forms of respiratory support, a significant number of preterm newborns, especially those born before 28 weeks of gestation require mechanical ventilation through an endotracheal tube. Although life saving, this is associated with numerous complications, including ventilator-induced lung injury (VILI) and bronchopulmonary dysplasia (BPD). VILI may result from multiple factors such as inconsistency in tidal volume delivery (too little causes atelectasis, too much causes overdistension and stretch injury), lack of synchronization between mechanical and spontaneous breaths; and direct injury to immature lungs by a variety of mechanisms such as inflammation and oxidative stress (1).

Concern about VILI has led to the introduction of newer strategies of ventilation, which are based on sound physiologic principles. Clinicians must familiarize themselves with these newer techniques, both from the viewpoints of clinical application and future research, especially as the scientific evidence for the safety and efficacy is still incomplete (2).

Because these forms of ventilation were not previously available to treat the neonatal population, this often causes confusion in light of the plethora of differing nomenclature and acronyms. To understand this clearly, it is advisable to use a simple hierarchical classification of ventilatory modes and modalities (3).
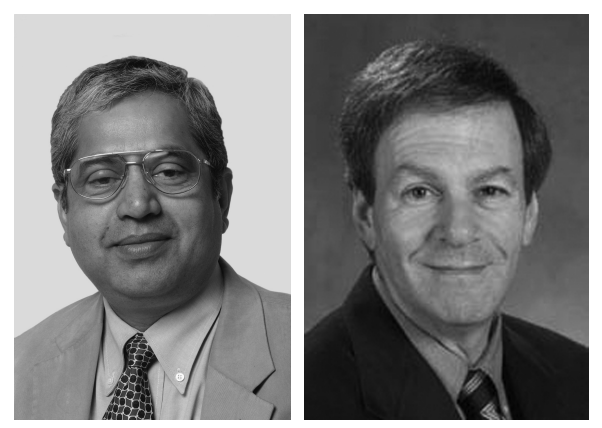

\section{CLASSIFICATION OF VENTILATION}

Ventilation can be classified by the variables that are 'controlled' or 'targeted' and cannot change, as well as 'phase' variables that are changeable and can be used to create individual breath types to suit specific pathophysiology.

\section{Control variables}

Parent mode: At any one time, the ventilator can control only pressure, volume, or flow. However, the same machine (especially the newer generation ventilators) can be used in different control modes at different times. As volume is the integral of flow, volume and flow controlled ventilators are actually the same.

\section{Phase variable}

Daughter modes: Both pressure and volume-controlled ventilation can be delivered in a variety of ways by creating different breath types. Each breath has four components (phases), and these can be created or modified using different variables. Thus, pressure, volume, flow, or time can be used as phase variables to trigger (initiate), limit (gas flow during inspiration), and cycle (cause inspiration to end) (Fig. 1). For example, in traditional pressure-limited, timecycled ventilation, pressure is used to limit the inspiratory flow and time is used to cycle inspiration into expiration (and vice versa). In contrast, in pressure support ventilation, 


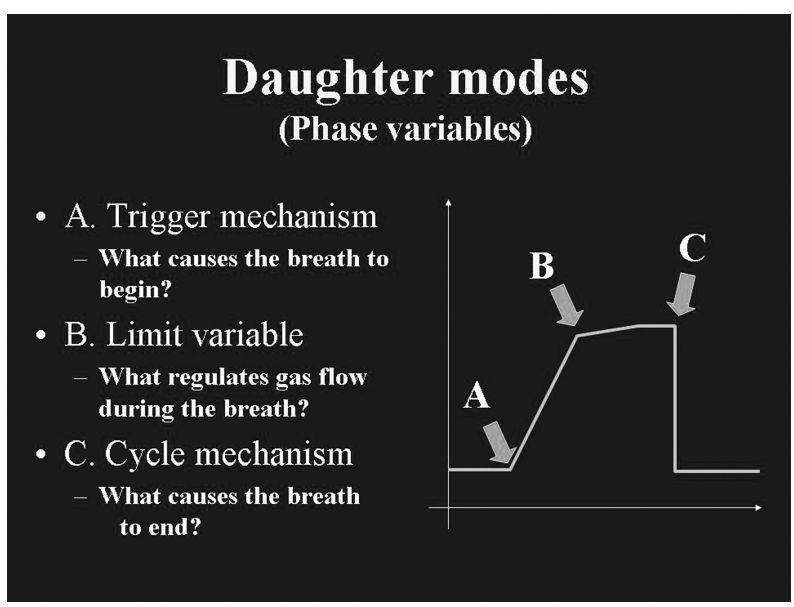

Figure 1 Different phases of a mechanical breath (trigger, limit and cycle) which can be changed using different variables such as pressure, time or flow.

inspiratory flow, rather than time, is used to cycle inspiration into expiration.

\section{PRESSURE-TARGETED MODALITIES OF VENTILATION}

Examples of pressure-targeted ventilation include traditional time-cycled pressure-limited ventilation (TCPLV), flow-cycled pressure-limited ventilation, pressure control ventilation (PCV) and pressure support ventilation (PSV) (4). What all of these have in common is a fixed pressure limit that the ventilator will not exceed. However, the delivery of tidal volume depends primarily on the patient's lung compliance. Thus, at a given pressure, tidal volume delivery will be lower when the lungs are stiff, and when compliance improves, the same amount of pressure will deliver a larger tidal volume. This has implications for clinical practice, as there is a potential for overdistension of the lungs in the face of improving compliance. This requires clinical decision making and ventilatory adjustment either manually or by using a computer-controlled algorithm as in some ventilators. Another distinguishing feature between different types of pressure-limited modalities is how the gas flow is delivered to the lungs. For example, in TCPLV inspiratory time and flow rates are fixed and chosen by the clinician. In contrast, flow-cycling results in variable inspiratory time (controlled by the patient). PCV and PSV also have variable inspiratory gas flow, which is proportional to patient effort.

\section{VOLUME-CONTROLLED VENTILATION}

In this form of ventilation, the clinician selects a specific tidal volume to be delivered by the ventilator irrespective of the state of lung compliance or the pressure required (5). To do this, the machine automatically adjusts the pressure to deliver the set tidal volume. Thus, when the lungs are stiff, a higher pressure is generated to deliver the set tidal volume, and as lung compliance improves, the same tidal volume is delivered by auto-weaning of pressure (Fig. 2).

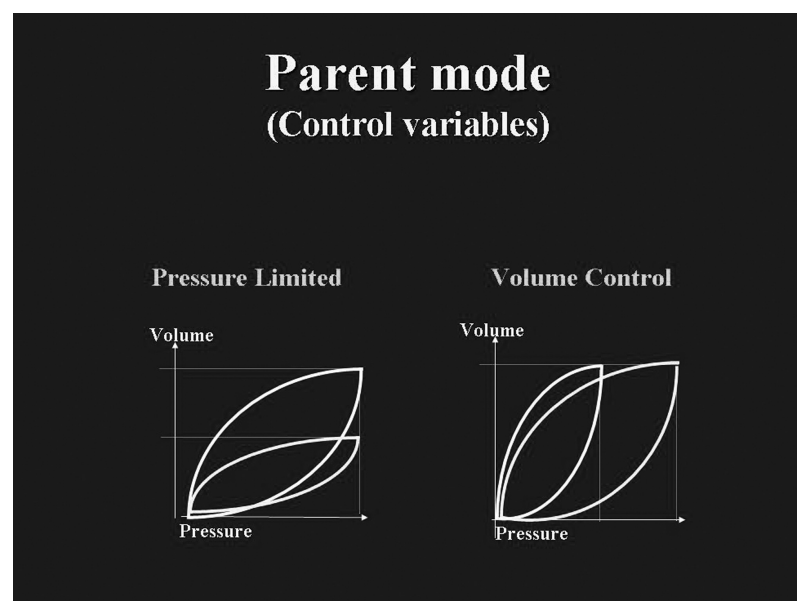

Figure 2 Difference between control mechanism of pressure and volume ventilation.

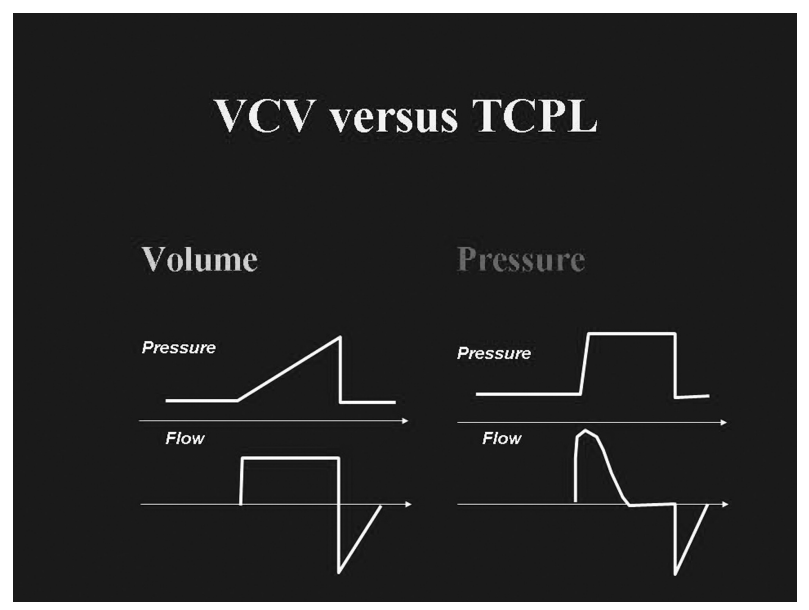

Figure 3 Difference between inspiratory gas flow pattern of volume and pressure-controlled ventilation.

Therefore, an advantage of volume-controlled ventilation over pressure-limited ventilation is that it responds to changes in pulmonary compliance. Volume-controlled ventilation also differs from pressure-limited ventilation in the way the inspiratory flow is delivered to the patient. In pressure-limited ventilation, the inspiratory flow rapidly accelerates and decelerates, resulting in rapid filling of the lung early in inspiration. In contrast, volume-controlled ventilation produces a square flow waveform, in which alveolar filling is slow and peak pressure and volume are achieved at the end of inspiration (Fig. 3). In other words, pressure-targeted modalities are 'front-end loaded' and may be more effective in treating stiff, atelectatic lungs, whereas in the volume-controlled modality, it is 'back-end loaded' and might work better in high lung volume states or where disease is more heterogeneous. Despite different inspiratory flow patterns, both pressure-limited ventilation and volume-controlled ventilation can be provided as intermittent mandatory ventilation, synchronized intermittent mandatory ventilation, and assist-control ventilation. 


\section{HYBRID FORMS OF MECHANICAL VENTILATION}

Attempts have been made to combine the desirable features of both pressure-targeted and volume-controlled ventilation resulting in a number of hybrid modalities. These include volume guarantee (VG), pressure regulated volume control (PRVC), and volume assured pressure support (VAPS). VG and PRVC are primarily pressure-targeted modes of ventilation but involve computerized servo-controlled ventilation, in which the ventilator has an algorithm that adjusts the rise and fall of pressure to produce tidal volume delivery based on the previous breaths. On the other hand, VAPS makes within-the-breath (intrabreath) adjustment of pressure and/or inspiratory time, until the desired volume has been provided.

\section{Volume guarantee ventilation}

Volume guarantee ventilation, available on Draeger Babylog ${ }^{\circledR} 8000$ ventilators, can be best described as a double- or dual-loop-synchronized mode that ventilates with a time-cycled pressure-limited breath type but allows the pressure to be adjusted, using microprocessor technology, to deliver a tidal volume in the range set by the clinician. The auto-feedback method 'guarantees' tidal volume based on an average of exhaled tidal volume from preceeding breaths. The starting tidal volume target is usually $4-6 \mathrm{~mL} / \mathrm{kg}$. The maximum pressure limit is set about $20 \%$ above the pressure needed to deliver this tidal volume (6).

Potential advantages of volume guarantee include less risk of volutrauma, as clinician-set tidal volume is not exceeded when lung compliance improves, reduced peak pressures when the baby makes significant contribution to tidal volume (thus reducing chances of barotrauma) more stable tidal volume delivery, and auto-weaning of peak inspiratory pressure, thus reducing barotrauma.

The feedback loop, however, may have limitations. As adjustments to PIP are made in small increments to avoid overcompensation, and are based on the exhaled tidal volumes, the delivered tidal volume cannot compensate for large breath-to-breath fluctuations (such as with large leaks). Moreover, as catch up adjustments in pressures occur every few breaths, it may not work if the ventilatory rate is set at low levels, for example, in SIMV at low rates.

\section{Pressure regulated volume control (PRVC)}

PRVC is another mode of ventilation, which attempts to combine the benefits of pressure-limited and volume controlled ventilation. This is available on the Servo 300A and the Servo-i ${ }^{\circledR}$ ventilators (Maquet). It is a flow-cycled modality that offers the variable flow rate of PCV with a targeted tidal volume. Like volume guarantee, PRVC is also a form of closed loop ventilation in which pressure is adjusted according to tidal volume delivered. The new Servo-i ventilator features wye-sensor measurements ensuring more accurate delivery of set tidal volume.

The clinician sets a target tidal volume and the maximum pressure to deliver the tidal volume. The microprocessor of the ventilator attempts to use the lowest pressure with a decelerating flow waveform to deliver the set tidal volume. The first breath is delivered at $10 \mathrm{~cm}_{2} \mathrm{O}$ above peak end expiratory pressure (PEEP) and is used as a test breath to enable the microprocessor to calculate the pressure needed to deliver the set tidal volume based on the patient's compliance. The next three breaths are delivered at a pressure of $75 \%$ of the calculated pressure needed. If targeted tidal volume is not delivered, the inspiratory pressure is increased by $3 \mathrm{~cm} \mathrm{H}_{2} \mathrm{O}$ for each breath until the desired tidal volume is reached. If targeted tidal volume is exceeded, the inspiratory pressure is decreased by $3 \mathrm{~cm} \mathrm{H}_{2} \mathrm{O}$. Inspiratory pressure is regulated by the ventilator between PEEP and $5 \mathrm{~cm}$ $\mathrm{H}_{2} \mathrm{O}$ below the clinician-set upper pressure limit. In PRVC, the pressure is adjusted on the average of the previous four breaths, so variations in delivered tidal volume could still occur.

\section{Volume assured pressure support ventilation (VAPS)}

This mode is available on the VIP Bird Gold ${ }^{\circledR}$ infant/ pediatric ventilator and can be best described as variable flow volume ventilation, which blends pressure support ventilation and volume-controlled ventilation. A guaranteed tidal volume delivery is provided with each breath. Each breath starts as a variable flow pressure support breath. The ventilator will measure the delivered tidal volume when the inspiratory flow has decelerated to a minimum set level. If the delivered tidal volume equals or exceeds the set tidal volume, the pressure support breath is allowed to continue and is flow-cycled. If the targeted tidal volume is not achieved, the breath changes to a volume-controlled breath with a constant flow waveform and inspiration is continued until the set tidal volume is delivered.

\section{PATIENT TRIGGERED (SYNCHRONIZED) VENTILATION}

Neonatal ventilation has traditionally been accomplished using intermittent mandatory ventilation (IMV); its major drawback is the development of asynchrony, whereby the ventilator cycles at a programmed rate and the patient breathes independently, sometimes with and sometimes against the mechanical breaths. Asynchrony has been shown to result in adverse physiological consequences, including inconsistent tidal volume delivery, insufficient gas exchange, air leaks, and increased work of breathing. Other organ systems may also be involved, including an association with intraventricular haemorrhage.

Synchronized or patient-triggered ventilation uses a patient-derived signal to initiate a mechanical breath. The signal is a surrogate of spontaneous breathing and consists of either a change in airway flow or pressure, abdominal movement, or thoracic impedance. A key to successful triggered ventilation is a short response time (trigger delay) and rapid delivery of gas to the proximal airway. The inspiratory flow signal may also be used to terminate (or cycle) a breath, thus fully synchronizing the baby and the ventilator during both inspiratory and expiratory phases. This is referred to as flow-cycling (7).

Examples of patient-triggered ventilation include synchronized intermittent mandatory ventilation (SIMV), assist/ control (A/C) ventilation and PSV. In SIMV, the ventilator 
will attempt to time the delivery of mechanical breaths to the onset of a spontaneous breath occurring in the 'timing window' (if the trigger threshold is met) or are delivered at the rate set by the operator if the patient effort is inadequate or absent. Between the mechanical breaths, the patient-breaths spontaneously, supported only by the baseline pressure. In $\mathrm{A} / \mathrm{C}$ ventilation, mechanical breaths are patient-initiated (assisted) if the patient's effort exceeds the trigger threshold, or ventilator-delivered (controlled) if the patient is unable to trigger the ventilator or fails to breathe above the control rate. If the patient is consistently breathing above the control rate, lowering the control rate has no effect on the mechanical rate. A/C has an advantage over SIMV in that it supports every breath that meets the trigger threshold (Fig. 4), thereby providing adequate minute ventilation at the minimal work of breathing. $\mathrm{A} / \mathrm{C}$ can be either time- or flow-cycled, but only the latter produces both inspiratory and expiratory synchrony. Flow-cycling terminates inspiration at a percentage of peak flow rather than time. PSV utilizes flow-cycling to achieve total synchrony as in flow-cycled $\mathrm{A} / \mathrm{C}$, but in addition it has variable inspiratory flow to enhance synchrony, comfort, and endurance. PSV provides an inspiratory pressure 'boost' to spontaneous breaths to help overcome the work of breathing imposed by the narrow lumen endotracheal tube and ventilator circuit dead space. PSV was designed to support spontaneous breathing during weaning from mechanical ventilation. Although it resembles flow-cycled A/C, PSV does not have a control (back up) rate and is often used in conjunction with SIMV as a 'safety' maneuver until reliable respiratory drive is observed (8).

Proportional assist ventilation (PAV) is an adaptive form of mechanical ventilation in which the inspiratory pressure is determined by the elastic and resistive properties of the lung. In PAV, the ventilator is continuously sensitive to respiratory effort, adjusting the assist pressure in a proportional and ongoing fashion. This may achieve near perfect synchrony between the ventilator and spontaneous breathing, with relief from disease-related increased mechanical work of breathing. The preliminary results are encouraging but further evaluation may help to define the role of this technique (9).

\section{Clinical studies}

As these modes and modalities of ventilation were introduced to the neonatal population only recently, there have been only a few clinical trials to compare their safety and efficacy. There are two published randomized controlled trials that evaluated volume-controlled ventilation. In the first study, 50 infants weighing $1200 \mathrm{~g}$ or more were randomized to either volume-controlled (VCV) or TCPLV. The VCV group reached the pre-determined success criteria faster and had a shorter mean duration of ventilation. There was a trend towards a reduced incidence of intraventricular haemorrhage and BPD in the VCV group (10). In the second study, 109 infants weighing between 600 and $1500 \mathrm{~g}$ and with a gestational age of 24 to 31 weeks, who had respiratory distress syndrome, were randomized. There was no difference in the time to reach the success criteria or total duration of venti-
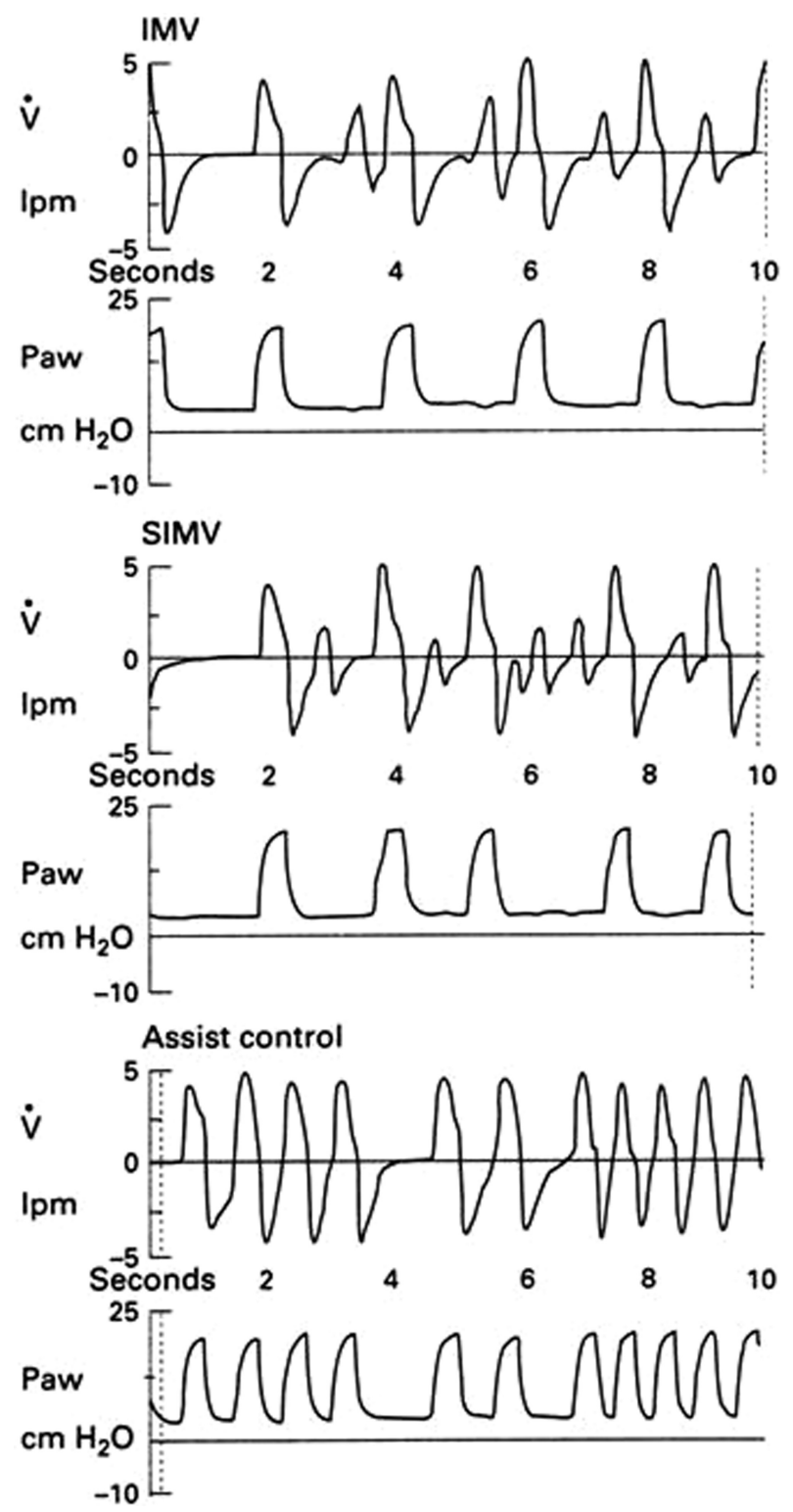

Figure 4 Flow and pressure waveforms for intermittent mandatory ventilation (IMV), synchronized intermittent mandatory ventilation (SIMV) and assistcontrol ventilation (A/C). During IMV (top), note mechanical breaths (larger flow waveforms) delivered at fixed intervals, set by the clinician. Spontaneous breaths may occur between mechanical breaths but may not generate significant positive pressure. During SIMV (middle), spontaneous patient effort above a set threshold can trigger a mechanical breath if it occurs within a 'timing window'. In between, spontaneous breaths can occur like IMV. During assist-control (A/C, bottom), each spontaneous breath that exceeds the trigger threshold is augmented by machine-delivered breath. Thus, each breath is identical and rate will vary according to a patients own rate. (with permission from Arch Dis Child, Sinha S K, Donn SM. Weaning from assisted ventilation:art or science? Arch Dis Child Fetal and Neonatal Edition. 2000;83(1):F64-70).

lation. However, sub-group analysis showed faster weaning in the VCV group of babies weighing $<1000 \mathrm{~g}$ ( $21 \mathrm{vs.} 58 \mathrm{~h}$; $\mathrm{p}=0.03)$ (11). A masked follow-up study of these infants at a median post-conceptional age of 22 months showed a continued trend towards better survival and clinical respiratory outcomes in the babies who had received VTV. 
The incidence of severe disability was also no higher in the volume group, but the study was not powered to address this outcome (12).

Volume guarantee ${ }^{\mathrm{TM}}(\mathrm{VG})$ is the most widely used method of providing volume-targeted ventilation but yet the least studied in clinical trials. Of the published trials of VG, most are in form of small crossover trials relating to endpoints based on short-term physiological changes. There is a lack of published data about the relevant long-term clinical outcomes. Cheema and Ahluwalia first investigated the feasibility and efficacy of VG in 40 premature newborn infants (mean birth weight $1064 \mathrm{~g}$, gestation 27.9 weeks) with respiratory distress syndrome, in a 4-h crossover trial. They found that the mean peak inspiratory pressure and mean airway pressure using VG with either synchronous intermittent positive pressure ventilation (SIPPV) or synchronous intermittent mandatory ventilation (SIMV) was lower than that with either SIPPV or SIMV alone. No untoward effects were noted during the study period. VG as used in this study seemed to be a feasible ventilation modality for neonatal patients and achieved equivalent gas exchange using statistically significant lower peak airway pressures both during early and recovery stages of RDS (13).

In another study, Herrera compared the effects of SIMV with VG and conventional SIMV on ventilation and gas exchange in a group of very low birth weight infants recovering from RDS. Nine infants were studied for two consecutive 60 -min intervals in a crossover trial. The authors concluded that VG led to automatic weaning of mechanical support and the enhancement of spontaneous respiratory effort during short-term use. The study was, however, based on a shortterm observation period in relatively stable babies. There was an upward trend in the transcutaneous $\mathrm{pCO}_{2}$ levels in the infants given VG (14).

Abubakar and Keszler in a short crossover trial, showed that breath-to-breath tidal volume variability was significantly reduced in VG compared to assist-control, SIMV, or pressure support ventilation alone (15). In another randomized trial by the same authors comparing $\mathrm{A} / \mathrm{C}$ to $\mathrm{A} / \mathrm{C}$ plus VG, the VG group maintained tidal volume and $\mathrm{PCO}_{2}$ within a target range more consistently. The authors concluded that VG reduced hypocarbia and excessive large tidal volume (16). Next, they compared the effect of A/C plus VG with SIMV plus VG in a short crossover trial. Infants in the SIMV plus VG group had higher variability of tidal volume and increased work of breathing (17). A more recent study has shown that VG is feasible in the initial stabilization of infants $>25$ weeks of gestation, and that it halves the incidence of hypocarbia compared to SIMV (18).

There have been two randomized controlled trials of PRVC. Although a subgroup analysis of infants $<1000 \mathrm{~g}$ in the first trial showed a reduction in the duration of ventilation in the PRVC, there was no difference in short-term survival, duration of ventilation or the incidence of BPD (19). Another randomized controlled trial compared PRVC with SIMV in a group of 213 ventilated infants found no difference in short-term survivors, duration of ventilation, or the incidence of chronic lung disease (20).
A Cochrane review of volume-targeted ventilation versus pressure-targeted ventilation, which included 178 infants, showed a significant reduction in duration of ventilation, and the rates of pneumothorax and severe intraventricular haemorrhage in babies treated with volume-targeted ventilation. The authors also reported a trend for reduction in the incidence of BPD [RR $=0.34$ (0.11 to 1.05)] favoring volume-targeting (21). An update of the review including the trial of Singh et al. is awaited.

Unfortunately, clinical trials on PTV have thus far been disappointing. Most of the trials have been under-powered to measure the impact on clinically relevant outcomes. A large open trial by Baumer et al., enrolling over 900 babies, did not show any advantage of PTV over IMV, (22) but this trial was rightly criticized for its methodological inadequacies (23). Unfortunately, this trial skews a meta-analysis of PTV, despite the fact that all of the other trials were positive (24). The concept of PTV makes good physiological sense but additional investigation is warranted.

\section{OPTIMIZING MECHANICAL VENTILATION OF THE PRETERM NEWBORN}

Choosing the proper strategy based upon the basic principles of mechanical ventilation should optimize pulmonary gas exchange and begin to reduce lung injury. RDS is a dynamic condition where the underlying pathophysiology is constantly changing, either as a part of the natural progression or in response to treatment. Therefore, it is necessary to adjust the ventilatory strategies to match the changing disease status. This can be facilitated by use of real-time pulmonary graphic monitoring, which provides breath-tobreath feedback on the interaction between the ventilator and the patient. It allows for the customization or 'fine tuning' of ventilation for the individual baby. Monitoring of pulmonary mechanics and waveforms also enables detection of events such as hyperinflation and gas trapping before they become clinically evident (25). These techniques can be helpful for determining the optimal positive endexpiratory pressure, adjusting the gas flow, and judging synchrony. Strategic ventilation aimed to correct the underlying pathophysiology seems likely to reduce BPD but unlikely to eliminate it completely, as factors other than VILI are also involved and they require individual attention. There is also a growing interest in the application of non-invasive forms of artificial respiratory support such as CPAP, but caution is required, especially for its use in very preterm and smaller babies as an initial treatment for respiratory failure.

What, then, is the ideal mode of ventilation? The ideal mode is one that provides and maintains adequate and consistent tidal volume delivery and minute ventilation at low airway pressures. It must also be able to respond quickly to rapid or unpredictable changes in patient demand or pulmonary mechanics. It should provide the lowest possible work of breathing for the baby. It is now increasingly being recognized that it is not the pressure but the amount of gas in the lungs, which is responsible for ventilator-related lung injuries and this has led to increasing popularity of volumetargeted ventilation in recent years. This has been possible 
because delivered tidal volumes can now be measured at the proximal airway. Optimal volume delivery may be a key factor in avoiding VILI in small preterm infants.

What is the ideal ventilator? The ideal ventilator is one that satisfactorily achieves the goals of mechanical ventilation. It should provide a variety of modes and modalities that can meet the requirements of even the most challenging pulmonary diseases. It must have monitoring capabilities to assess patient and ventilator interaction and performance. Although the choice of different ventilation modes are now available to the clinician, none of them comes without its own limitations and advantages. It is important to understand the mechanisms of different forms of ventilation so as to use the appropriate style for a specific situation rather than a single form of ventilation for all respiratory problems needing assisted ventilation. There is also a need for further randomized controlled trials comparing different modalities of ventilation targeted to a specific underlying pathology. Until then, it would be premature to justify the superiority of any particular modality over another mode of ventilation.

\section{SUMMARY}

Although life saving, mechanical ventilation can cause complications such as VILI and BPD in very preterm babies. VILI is multi-factorial. There has been introduction of a number of newer techniques of mechanical ventilation, which are aimed to reduce such complications. These are based on sound physiologic principles and clinicians should familiarize themselves with these advances. The consideration that volutrauma rather than barotrauma causes more VILI, has resulted in a growing interest in volume-targeted forms of ventilation. Although the long-term efficacy of volume-targeted ventilation against conditions such as BPD is not yet established, the results of the published trials of VCV and meta analysis show benefits by way of reduced duration of ventilation and pneumothoraces. Although shortterm, these clinical outcomes are still relevant.

\section{References}

1. Donn SM, Sinha SK. Minimising ventilator induced lung injury in preterm infants. Arch Dis Child Fetal Neonatal Ed 2006; 91: F226-30.

2. Donn SM, Sinha SK. Can mechanical ventilation strategies reduce chronic lung disease? Semin Neonatal 2003; 8: 441-8.

3. Carlo WA, Ambalavanan N, Chatburn RL. Classification of mechanical ventilation devices. In Donn SM, Sinha SK editors. Manual of neonatal respiratory care. 2nd ed. Philadelphia: Mosby Elsevier, 2006; 74-80.

4. Gupta S, Sinha SK. Newer modalities of mechanical ventilation in the extremely premature infants. Paediatr Child Health 2007; 17: 37-42.

5. Sinha SK, Donn SM. Volume-controlled ventilation: variations on a theme. Clin Perinatol 2001; 28: 547-60.

6. Keszler M, Abubakar KM. Volume guarantee ventilation. Clin Perinatal 2007; 34: 107-16.
7. Donn SM, Sinha SK. Newer modes of mechanical ventilation for the neonate. Curr Opin Pediatr 2001; 13: 99-103.

8. Sarkar S, Donn SM. In support of pressure support ventilation. Clin Perinatol 2007; 34: 117-28.

9. Schulze A, Bancalari E. Proportional assist ventilation in infants. Clin Perinatol 2001; 23: 561-78.

10. Sinha SK, Donn SM, Gavey J, McCarty M. Randomised trial of volume controlled versus time cycled, pressure limited ventilation in preterm infants with respiratory distress syndrome. Arch Dis Child Fetal Neonatal Ed 1997; 77: F202-5.

11. Singh J, Sinha SK, Clarke P, Byrne S, Donn SM. Mechanical ventilation of very low birth weight infants: is volume or pressure a better target variable? J Pediatr 2006; 149: 308-13.

12. Singh J, Alsop E, Sinha SK, Gupta S. Volume control versus pressure limited ventilation in very low birth weight infants: long term follow up of a randomised controlled trial. E-PAS 2007; 616090.2.

13. Cheema IU, Ahluwalia JS. Feasibility of tidal volume-guided ventilation in newborn infants: a randomised, crossover trial using the volume guarantee modality. Pediatrics 2001; 107: 1323-8.

14. Herrera CM, Gerhardt T, Claure N, Everett R, Musante G, Thomas C. Effects of volume-guaranteed synchronized intermittent mandatory ventilation in preterm infants recovering from respiratory failure. Pediatrics 2002; 110: 529-33.

15. Abubakar KM, Keszler M. Patient-ventilator interactions in new modes of patient-triggered ventilation. Pediatr Pulmonol 2001; 32: 71-5.

16. Keszler M, Abubakar KM. Stability of tidal volume and incidence of hypocarbia. Pediatr Pulmonol 2004; 38: 240-5.

17. Abubakar KM, Keszler M. Effect of volume guarantee combined with assist/control vs synchronized intermittent mandatory ventilation. J Perinatol 2005; 25: 638-42.

18. Cheema IU, Sinha AK, Kempley ST, Ahluwalia J. Impact of volume guarantee ventilation on arterial carbon dioxide tension in newborn infants: a randomized controlled trial. Early Hum Dev 2007; 83: 183-9.

19. Piotrowski A, Sobala W, Kawczynski P. Patient-initiated pressure regulated volume control ventilation compared with intermittent mandatory ventilation in neonates: a prospective randomised study. Intensive Care Medi 1997; 23: 975-81.

20. D'Angio CT, Chess PR, Kovacs SJ, Sinkin R, Phelps D, Kendig J. Pressure-regulated volume control ventilation vs synchronised intermittent mandatory ventilation in very low-birth-weight infants. Arch Pediatr Adolesc Med 2005; 159: 868-75.

21. McCallion N, Davis PG, Morley CJ. Volume-targeted versus pressure-limited ventilation in the neonate. Cochrane Database Syst Rev 2005: CD003666.

22. Baumer JH. International randomized controlled trial of patient triggered ventilation in neonatal respiratory distress syndrome. Arch Dis Child Fetal Neonatal Ed 2000; 82: F5-10.

23. Donn SM, Greenough A, Sinha SK. Patient triggered ventilation. Arch Dis Child Fetal Neonatal Ed 2000; 83: F225-6.

24. Greenough A, Milner AD, Dimitriou G. Synchronized mechanical ventilation for respiratory support in newborn infants. Cochrane Database Syst Rev 2004: CD000456.

25. Becker MA, Donn SM. Real-time pulmonary graphic monitoring. Clin Perinatol 2007; 34: 1-17. 